\title{
Transient Osteoporosis of the Hip and Hyperbaric Oxygen Therapy: A Report of Two Cases
}

\author{
Serdar KESIKBURUN'1, Ayça URAN'1, Yasin DEMIR', Ümüt GÜZELKÜÇÜK', Serkan ERGÖZEN², Arif Kenan TAN \\ 'Department of Physical Medicine and Rehabilitation, Gülhane Military Medical Academy, Turkish Armed Forces Rehabilitation Center, Ankara, Turkey \\ ${ }^{2}$ Department of Underwater and Hyperbaric Medicine, Gülhane Military Medical Academy, Ankara, Turkey
}

\begin{abstract}
Transient osteoporosis of the hip is not a common disease. It is self-limited and characterized by the sudden onset of hip pain. It affects mostly middle-aged men and women in the third trimester of pregnancy and early postpartum period. The treatment consists of rest, decrease in weight bearing on hip, analgesics, and anti-resorptive drugs. We present two patients who were diagnosed with transient osteoporosis of the hip and had reduction in symptoms with hyperbaric oxygen therapy. In this report, we aimed to discuss the clinical features of the disease and the role of in its treatment.

Keywords: Transient osteoporosis, hip pain, hyperbaric oxygen therapy
\end{abstract}

\section{Introduction}

Transient osteoporosis is a self-limited clinical picture leading to the sudden onset of joint pain after the formation of local osteopenia and having a course of spontaneous recovery (1). Bone marrow edema in the femur head and demineralization occur in transient osteoporosis of the hip ( $\mathrm{TOH})$. Its etiology is not exactly known. It affects mostly middle-aged men and women in the third trimester of pregnancy or in the early postpartum period (2). The treatment includes rest, decreasing weight bearing on the hip, analgesics, and anti-resorptive drugs. Hyperbaric oxygen (HBO) therapy is based on the application of $100 \%$ oxygen to the whole body in pressure levels higher than atmospheric pressure by way of inhalation. It has been considered that HBO therapy can be used in $\mathrm{TOH}$ treatment with its effects of reducing intraosseous edema with vasoconstriction and increasing bone repair $(3,4)$. In this paper, we present two cases who were admitted to our outpatient clinic with hip pain, diagnosed with transient osteoporosis of the hip, and applied HBO therapy.

\section{Case Reports}

\section{Case 1}

A 33-year-old female patient was admitted to our center with the complaint of pain in the left hip on the $27^{\text {th }}$ day after delivery. The history of the patient revealed that she had been primipara and had delivered by cesarean at term. The patient stated that the hip pain had begun a few days after the delivery, its intensity had gradually increased, and the pain had prevented her walking. In her physical examination, the range of motions of the left hip joints was normal but heavily painful. The range of motions of the right hip and waist was normal and painless. Straight leg raise and sacroiliac stretching tests were negative on both sides. No pathological finding was encountered during her neurological examination. There was no abnormality in the 

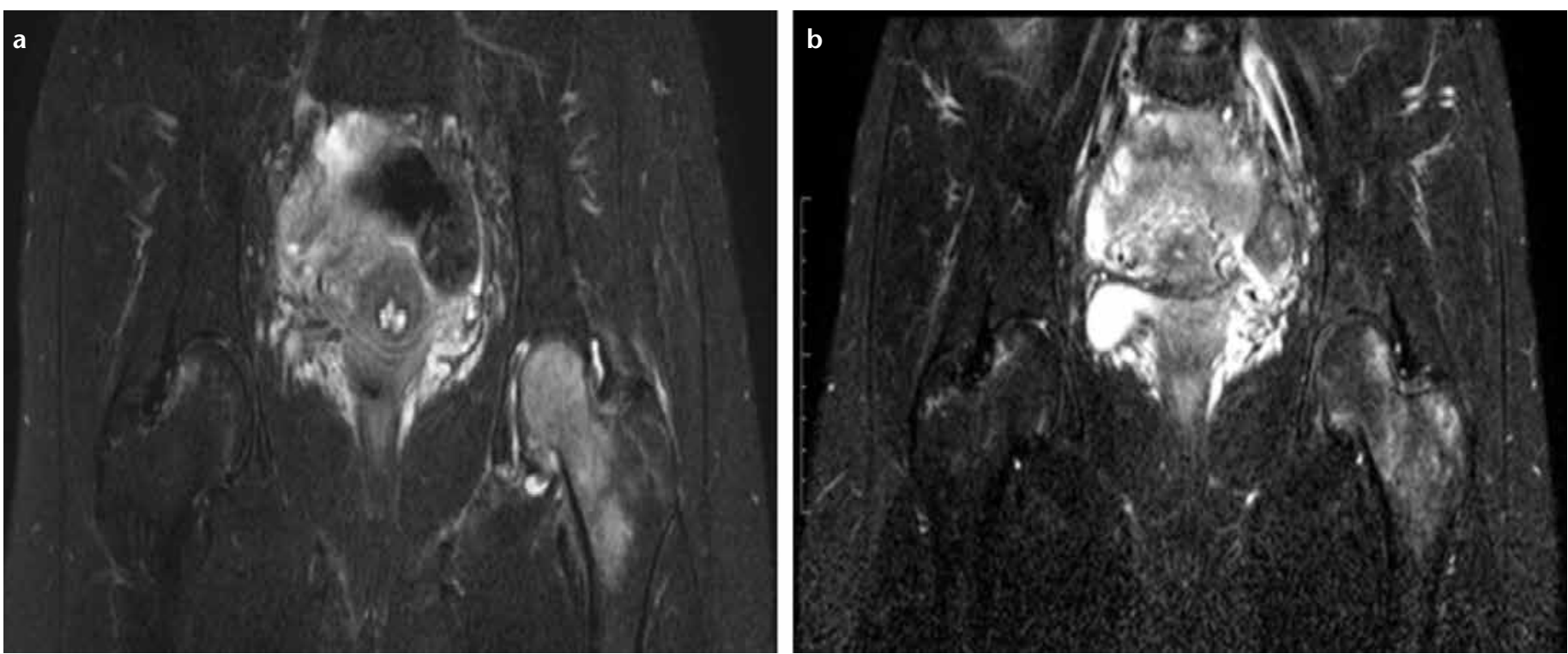

Figure 1. a,b. In MRI of the left hip, (a) hyperintense view of diffuse bone marrow edema in the left femoral head and neck and in the subtrochanteric region in T2-weighted sections, and in MRI taken in the control examination 2 months after the hyperbaric oxygen therapy and (b) a regression in bone marrow edema are observed
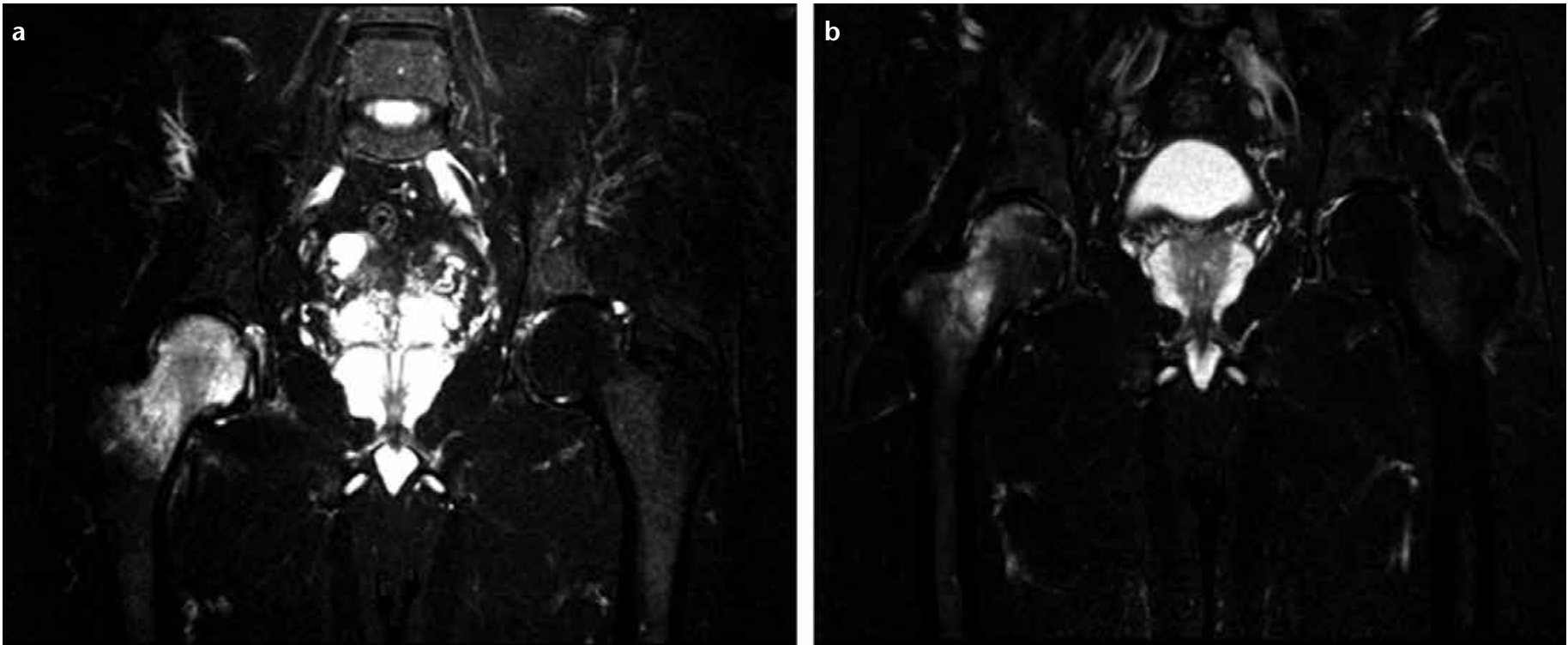

Figure 2. a,b. In T2-weighted sections of MRIs taken before (a) and 2 months after (b) hyperbaric oxygen therapy, a decrease in bone marrow edema in the right femoral head and neck is observed

laboratory investigations, including hemogram, sedimentation, C-reactive protein (CRP), and biochemistry parameters. The patient underwent hip radiography, and the radiograph findings were evaluated to be normal. In the T2-weighted sections of magnetic resonance imaging (MRI) of the left hip, hyperintense view of diffuse bone marrow edema in the left femoral head and neck and in the subtrochanteric region and mild hip effusion were observed (Figure 1). The patient was diagnosed with left $\mathrm{TOH}$ as a result of these findings. The patient was prescribed a single forearm crutch to decrease weight bearing on the left hip during ambulation. A home exercise program, including active range of motion exercises and isometric strengthening exercises for the muscles around the hip was administered to the patient. Because she was in the breast feeding period, drug therapy, except $500 \mathrm{mg}$ paracetamol in case of any requirement, was not administered. She consulted a physician from the Department of Underwater and Hyperbaric Medicine, and HBO therapy was planned. HBO therapy was administered in a pressurized room by breathing pure oxygen at 2.4 atmosphere absolute (ATA) via a mask for 120 min for five sessions a week (total 10 sessions). After the completion of the therapy 2 weeks later, the patient stated that there was a notable decrease in her pains. In her MRI 
taken in the control examination after 2 months, a regression was observed in her bone marrow edema in the left hip (Figure 1). The patient reported that her pains had decreased to a great extent, she did not have difficulty in walking, and she did not have to use forearm crutch. Written informed consent was obtained from the patient for the case report.

\section{Case 2}

A 52-year-old male patient visited our center with a complaint of sudden onset of localized pain in the right hip region. His history revealed that his pain increased with weight bearing, prevented his walking, and he could not perform his daily activities because of gradually increasing severity of pain. He had no history of any trauma, metabolic disease, or drug use. In the physical examination, it was detected that he could not do the joint motions in the right hip, particularly internal rotation movement, because of pain. No abnormality was found in the neurological examination. The results of his blood analyses, including hemogram, routine biochemistry, sedimentation, and CRP, were normal. The result of the direct radiography of the hip was also normal. MRI of the left hip was planned for the patient. MRI revealed diffuse bone marrow edema in the left femur head and neck (Figure 2). The patient was diagnosed with $\mathrm{TOH}$. Non-steroid anti-inflammatory drug (NSAID) therapy was initiated, and a single forearm crutch was prescribed for decreasing the weight on the healthy side. An exercise program, including range of motion and isometric strengthening exercises, was arranged. Because he did not significantly benefit from the applied therapies, bisphosphonate therapy and HBO therapy were recommended as alternative treatments to the patient. He rejected supplemental drug therapy but accepted HBO therapy. After the consultation of underwater and hyperbaric medicine, 10 sessions of HBO therapy were planned. The therapy was applied by breathing oxygen via a mask at 2.4 ATA for 120 min (as five sessions a week) in a pressurized room. After a 10-session therapy, a partial regression was observed in his symptoms. Therefore, his HBO therapy was completed in 20 sessions by prolonging for another 10 sessions. After the treatment, a remarkable decrease was observed in his pain. MRI, which was taken 2 months after the therapy, revealed a decrease in the bone marrow edema in the right hip (Figure 2). Written informed consent was obtained from the patient for the case report.

\section{Discussion}

Transient osteoporosis is a self-limited clinical picture that particularly affects the hip and sometimes the spine and ribs (1). Transient osteoporosis of the hip is rarely observed, and its etiology is not exactly known (2). It is accepted that it is not associated with trauma, and it is an idiopathic picture (5). TOH has been considered to be an early and reversible phase of avascular necrosis with venous return insufficiency. In another view, $\mathrm{TOH}$ has been evaluated as a kind of reflex sympathetic dystrophy. According to this view, the underlying mechanism is the prolonged or excessive activation of regional accelerating phenomenon in the bone triggered by painful stimulation caused by microfractures (2).
In $\mathrm{TOH}$, acute hip pain spontaneously initiating is observed. Pain can extend to the groin and thigh, and it increases with weight. Antalgic gait due to severe pain is observed in patients. In the physical examination, the range of motion is maintained, except advanced level of rotation movement, and joint motions are painful. Because $\mathrm{TOH}$ is a diagnosis for ruling out, the differential diagnosis of hip pain must be established. In differential diagnosis, inflammatory joint diseases, septic arthritis, primary or metastatic malignancies, pigmented villonodular synovitis, synovial chondromatosis, femoral neck stress fracture, and osteonecrosis must be evaluated. Laboratory findings are generally found to be normal; however, a slight increase in the urinary hydroxyproline, erythrocyte sedimentation rate, and serum alkaline phosphates and a slight decrease in calcitriol level can be observed in some patients (2). Imaging techniques are highly useful in diagnosis. Serious osteopenia and joint effusion occur in patients in a few weeks, and the results of direct radiographies in early stages of $\mathrm{TOH}$ may be found as normal (5). In direct radiographies, joint space, acetabulum, and subchondral bone are preserved; however, periarticular osteopenic changes are observed in the femoral head and neck between the $4^{\text {th }}$ and $8^{\text {th }}$ weeks (6). MRI of the hip is important for TOH diagnosis. Bone marrow edema that is viewed as hyperintense in T2-weighted sections and homogenously extends in the femoral head and neck suggests $\mathrm{TOH}$ (7). Characteristic bone marrow edema changes can appear $48 \mathrm{~h}$ after the onset of symptoms (2). In MRI, its differential diagnosis with avascular necrosis, which causes focal lesions in the subchondral region, must be made $(6,7)$. Radiological findings can continue to be observed even weeks after the improvement of symptoms (7). In our cases, diffuse bone marrow edema in femoral head and neck, which was observed in the hip MRI taken in early stage, was guiding, and no abnormal finding was detected in direct radiographies.

$\mathrm{TOH}$ is a disease with symptoms that spontaneously regress a few months after its occurrence $(2,5)$. The essential principle in treatment must be to reduce the pain of the patient at an early stage. Therefore, the patient must get rest and weight bearing must be restricted. Patients must be prescribed to use a walking stick or forearm crutch. Their duration of usage is unclear; however, it can be adjusted according to the severity of symptoms. In order to reduce pain, paracetamol, NSAID, and tramadol can be used. Moreover, various physical therapy modalities can also be utilized. For the prevention of joint motion and muscle strength loss that can occur with rest in patients, range of motion exercises and isometric strengthening exercises for the muscles around the hip are recommended (2). In the literature, there are data revealing that bisphosphonates decrease pain and shorten the duration of disease (8). lloprost therapy is another pharmacological agent, and its usage has been previously reported.

Hyperbaric oxygen therapy is the intermittent inhalation of $100 \%$ oxygen at a higher pressure than atmospheric pressure. In single or multiple hyperbaric rooms, patients breathe oxygen via a mask or hood. Oxygen inspiration at high pressure increases the amount of dissolved oxygen in plasma. High amount of oxygen in plasma elevates tissue oxygenation (9). 
$\mathrm{HBO}$ has been used in the treatment of $\mathrm{TOH}$ by considering that it might be useful $(3,4)$. The first mechanism of action of $\mathrm{HBO}$ in the treatment of $\mathrm{TOH}$ can believed to be vasoconstrictive effect. With the administration of $\mathrm{HBO}$, vasoconstriction occurs in the femoral head and tissue edema decreases. When edema disappears, intraosseous pressure decreases and venous drainage and microcirculation improve (10). This effect of $\mathrm{HBO}$ is similar to its usage in the treatment of early stage avascular necrosis of the hip. The increasing effect of HBO on the bone formation and repair can be demonstrated as another action of mechanism. It has been revealed that $\mathrm{HBO}$, which speeds up fracture healing, increases type 1 collagen formation from osteoblasts and alkaline phosphatase activity (11).

There is no clear algorithm showing the appropriate dose and session number of $\mathrm{HBO}$ in the treatment of $\mathrm{TOH}$. In our cases, HBO therapy was planned on the basis of the doses and durations of $\mathrm{HBO}$ therapy used in the treatment of avascular necrosis of the hip. In a study conducted by Reis et al. (10), 12 patients with stage- 1 avascular necrosis in the femoral head were administered $100 \%$ HBO therapy (2-2.4 ATA) for 90 min per session for 100 days, and it was found that MRI findings returned to normal in $81 \%$ of patients. In another study performed by Enrico et al. (12), 20 sessions of 100\% HBO therapy, each of which lasted $82 \mathrm{~min}$ at the level of 2.5 ATA, were administered to 20 patients with unilateral necrosis of the femoral head and pain was reduced at a significant ratio. In contrast, because of HBO therapy varying between 20 and 30 sessions, a significant improvement was observed in the range of joint motions. In our first case administered HBO therapy, the patient reported that her pain in the hip decreased at the beginning of sessions and a remarkable relief was observed after the completion of 10 sessions. Considering long-term HBO therapy in avascular necrosis of the hip in literature, an extra 10-session therapy was approved for the patient $(10,12)$, and after these additional 10 sessions of therapy, desired improvement was obtained in the patient. Further clinical studies evaluating the efficiency of HBO therapy in $\mathrm{TOH}$ and its implementation protocols are required.

One of the most important issues in the evaluation of new treatment modalities is undoubtedly the cost of treatment. In studies, HBO therapy is evaluated to be a cost-effective method that can be used for musculoskeletal disorders, particularly for the conditions in which oxygenation is impaired $(13,14)$. The use of $\mathrm{HBO}$ therapy in $\mathrm{TOH}$ can come into prominence, particularly in cases for which other alternative therapies cannot be applied. Besides that because there is no cost effectiveness study regarding the use of $\mathrm{HBO}$ in patients with $\mathrm{TOH}$, it is necessary to make related analyses in this area.

\section{Conclusion}

In patients without a history of trauma, but with a sudden onset of hip pain, the diagnosis of $\mathrm{TOH}$ must absolutely be considered. Appropriate clinical, laboratory, and imaging techniques must be used for the differential diagnosis. Conservative methods for pain in early stage must be applied in the treatment. HBO therapy can provide additional benefit for reducing $\mathrm{TOH}$ symptoms in this period.

Informed Consent: Written informed consents were obtained from the patients who participated in this case report.

Peer-review: Externally peer-reviewed.

Author contributions: Concept - S.K., A.U.; Design - A.U.,S.K.; Supervision - U.G., Resource - Y.D.; Data Collection and/or Processing - S.E.; Literature Search - S.E., Y.D.; Writing - S.K., A.U.; Critical Reviews - A.K.T.

Conflict of Interest: No conflict of interest was declared by the authors.

Financial Disclosure: The authors declared that this study has received no financial support.

\section{References}

1. Crespo E, Sala D, Crespo R, Silvestre A. Transient Osteoporosis. Acta Orthopaedica Belgica 2001;67:4.

2. Guzel R, Basaran S. Kalçanın Geçici Osteoporozu. Türk Fiz Tıp Rehab Derg 2009;55:41-5.

3. Mutluoglu M, Sonmez G, Sivrioglu AK, Ay H. There may be a role for hyperbaric oxygen therapy in transient osteoporosis of the hip. Acta Orthop Belg 2012;78:685-7.

4. Domachevsky L, Keynan Y, Militianu D, Goldenberg I, Adır Y. Transient Osteoporosis Associated With Hyperhomocystinemia: A Possible Role For Hyperbaric Oxygen Therapy. Undersea Hyperb Med 2004;31:275-9.

5. Demirkale I, Kılıçarslan K, Akkurt MO, Özdemir M. Geçici Osteoporoz. TOTBID Dergisi 2013;12:68-72. [CrossRef]

6. Balakrishnan A, Schemitsch EH, Pearce D, McKee MD. Distinguishing transient osteoporosis of the hip from avascular necrosis. Can J Surg 2003;46:187-92.

7. Grimm J, Higer HP, Benning R, Meairs S. MRI of transient osteoporosis of the hip. Arch Orthop Trauma Surg 1991;110:98-102. [CrossRef]

8. Emad Y, Ragab Y, El-Shaarawy N, Rasker JJ. Transient osteoporosis of the hip, complete resolution after treatment with alendronate as observed by mrı description of eight cases and review of the literature. Clin Rheumatol 2012;31:1641-7. [CrossRef]

9. Behnke AR, Saltzman HA. Hyperbaric oxygenation. N Engl J Med 1967;276:1423-9. [CrossRef]

10. Reis ND, Schwartz O, Militianu D, Ramon Y, Levin D, Norman D, et al. Hyperbaric oxygen therapy as a treatment for stage-I avascular necrosis of the femoral head. J Bone Joint Surg Br 2003;85:371-5. [CrossRef]

11. Kawada S, Wada E, Matsuda R, Ishii N. Hyperbaric hyperoxia accelerates fracture healing in mice. PLoS One 2013;8:e72603. [CrossRef]

12. Camporesi EM, Vezzani G, Bosco G, Mangar D, Bernasek TL, Hyperbaric oxygen theraphy in femoral head necrosis. J Arthroplasty 2010;25:118-23. [CrossRef]

13. Wang J, Li F, Calhoun JH, Mader JT, The role and effectiveness of adjunctive hyperbaric oxygen therapy in the management of musculoskeletal disorders. J Postgrad Med 2002;48:226-31.

14. Treweek S, James PB. A cost analysis of monoplace hyperbaric oxygen therapy with and without recirculation. I Wound Care 2006; 15:235-8. [CrossRef] 